\title{
AGN STATISTICS OF SIMULTANEOUS \\ RADIO AND GAMMA RAY OBSERVATIONS
}

\author{
A. MÜCKE, M. POHL AND G. KANBACH \\ MPE, Postfach 1603, D-85748 Garching, Germany \\ AND \\ P. REICH, W. REICH, R. SCHLICKEISER \\ MPIfR, Postfach 2024, D-53010 Bonn, Germany
}

In this paper we present first results of a statistical analysis of correlated variability behaviour of flat-spectrum radio quasars (FSRQ) on the basis of EGRET data and cm-wavelength monitoring data. We use EGRET observations obtained between April 1991 to September 1993 and multifrequency radio observations at $2.8 \mathrm{~cm}, 6 \mathrm{~cm}$ and $11 \mathrm{~cm}$ taken with the $100-\mathrm{m}$ Effelsberg Telescope parallel to the CGRO observations. In the following discussion the observed FSRQ which have not been detected by EGRET yet, are referred to as 'candidates' in contrast to the detected ones, called 'EGRET-sources'. The methods used in this paper are described in more detail in Mücke et al. (1996).

\section{The radio state of EGRET sources}

Our comparison of the $2.8 \mathrm{~cm}$ flux density distribution and the $2.8 \mathrm{~cm}$-to$6 \mathrm{~cm}$ spectral index distribution of EGRET sources to candidates is based on 15 highly variable $\left(\left|S_{\max }-S_{\min }\right| / S_{\min }<100 \%\right)$ EGRET-sources and 22 candidates. A total of 18 simultaneous flux and 14 spectral index measurements in the cm-radio and EGRET regime exist. Historical minima and maxima were used to define a linear scale of activity. The analysis is done with likelihood tests and Kolmogorov-Smirnov tests. The probability that the activity distributions of the candidates and EGRET-sources considered on long time scales are statistically identical is $48 \%$. However, at the time of an EGRET-detection the EGRET-sources show a more evenly distribution while the candidates and the monitored EGRET-sources have a higher chance to be detected at low activity. The distribution of the monitored 
EGRET-sources deviate from those simultaneously observed by $2.1 \sigma$, while the distribution of the EGRET-sources and candidates differ only by $0.7 \sigma$. Therefore, we conclude that $\gamma$-ray emission is accompanied by an enhanced radio activity. The spectral index distributions of EGRET-sources and candidates differ with a significance of $2.1 \sigma$. Indeed, for EGRET-sources an inverted spectrum seems to be more probable. This might also be true for simultaneously observed EGRET-sources.

2. Correlations between simultaneous measurements in the radio and $\gamma$-ray regime

Again we use the high-frequency $(2.8 \mathrm{~cm})$ radio data. We searched for linear correlations with a $\chi^{2}$-test using scaled data. No convincing linear correlation is found between the $\gamma$-ray flux and the radio spectral index, and between the $\gamma$-ray flux and the simultaneous $2.8 \mathrm{~cm}$ radio flux density. This is in contrast to the results of other authors (Dondi et al. 1995, Padovani et al. 1993, Salamon \& Stecker 1994) who found linear correlations between non-simultaneous observations of radio and $\gamma$-ray luminosities which should also be visible in terms of flux. Note, however, that flux-limited samples and the strong dependence of the luminosities on the redshift may simulate a linear correlation. An explanation of the non-correlation of simultaneous radio and $\gamma$-ray observations may be a time shift between the radio and $\gamma$-ray outburst.

It is found that the $\gamma$-ray spectrum tends to harden with increasing $\gamma$-ray flux. The $\chi^{2}$-fit of this correlation has a significance of $73 \%$, and a slope of zero can be ruled out with a probability of $10^{-5}$.

\section{EGRET detection versus radio state}

The characterization of the radio state of each source during an EGRETobservation was performed using 5 classes. The probability of an EGRETdetection compared to an EGRET-non-detection at the different radio states indicates that $\gamma$-rays are detected preferably when the radio light curve is increasing. FSRQ have been never seen by EGRET when they are in a decreasing radio state.

\section{References}

Dondi, L., Ghisellini, G. 1995, MNRAS , $273,583$.

Mücke, A., Pohl, M., Reich, P., et al. 1996, Proc. $3^{\text {rd }}$ Compton Symposium, A\&AS submitted.

Padovani, P., Ghisellini, G., Fabian, A.C., et al. 1993, MNRAS, 260, L21.

Reich, W., Steppe, H., Schlickeiser, R., et al. 1993, $A \& A, 273,65$.

Salamon, M.H., Stecker, F.W. 1994, ApJ, 430, L21. 